$\mathrm{DE}$

M E D I C I N A

T R O P I C A L

$\mathrm{DE}$

SÃO PAULO

JOURNAL OF THE SÃO PAULO INSTITUTE OF TROPICAL MEDICINE

${ }^{1}$ Hospital Universitario Mayor-Méderi, Bogotá, Colombia

2Universidad del Rosario, Escuela de Medicina y Ciencias de la Salud, Bogotá, Colombia

${ }^{3}$ Compensar EPS, Laboratorio Clínico, Bogotá, Colombia

${ }^{4}$ Universidad del Rosario, Escuela de Medicina y Ciencias de la Salud, Grupo de Estudios en Microbiología Traslacional y Enfermedades Emergentes, Bogotá, Colombia

Correspondence to: Carolina Firacative Universidad del Rosario, Escuela de Medicina y Ciencias de la Salud, Grupo de Estudios en Microbiología Traslacional y Enfermedades Emergentes, CII 63C \# 26-63, 111221, Bogota, Colombia Tel +5712970200 Ext 3404

E-mail: cfiracative@gmail.com

Received: 10 December 2019

Accepted: 7 April 2020

\section{Fatal fungaemia due to Cryptococcus albidus in an elderly diabetic woman presenting with pleural effusion}

\author{
Giovanni Rodríguez-Leguizamón ${ }^{(1,2}$, Eliana Coque-Burgos ${ }^{(1)} 3$, Omar \\ Espitia-Castro', Carolina Firacative ${ }^{(1)} 4$
}

\section{ABSTRACT}

This is a fatal case of fungaemia due to Cryptococcus albidus in an elderly woman with type 2 diabetes mellitus who presented pleural effusion as the only clinical presentation. Not only infections of the pleura are uncommon presentations of pulmonary cryptococcosis, but these infections due to non-C. neoformans species are extremely rare. This report places C. albidus on the growing number of disseminated mycosis-causing agents in diabetic patients.

KEYWORDS: Pleural effusion. Cryptococcus. Fungemia. Diabetes Mellitus, type 2. Elderly. Colombia.

\section{INTRODUCTION}

Cryptococcosis is an important opportunistic fungal infection that in the vast majority of cases is caused by Cryptococcus neoformans, a basidiomycetous encapsulated yeast that lives in diverse environmental niches. This mycosis, which is initially established in the respiratory tract after the inhalation of fungal cells (likely desiccated yeast or spores), presents most frequently as meningitis or meningoencephalitis, predominantly among HIV patients ${ }^{1}$. However, cryptococcosis can affect any tissue or organ of the human body and the risk of severe invasive or disseminated disease increases if the infection is left untreated and is highly dependent on the degree of immunosuppression of the patient ${ }^{2}$. To our knowledge, this report is the first to describe a fatal case of cryptococcemia, with pleural effusion as the only presentation, caused by a non-C. neoformans species, Cryptococcus albidus, in a non-HIV, but chronically ill, elderly female patient from Colombia. This case emphasizes the increasing risk for immunocompromised patients and those with underlying conditions, such as diabetes, to acquire systemic mycoses due to infrequent environmental pathogens.

\section{CASE REPORT}

An 85-year old woman with type 2 diabetes mellitus (T2DM) and arterial hypertension was admitted to a secondary-level hospital in Bogota on October 2, 2017 (day 0) with hyperglicemia. The patient presented with fatigue, polyuria, polydipsia and nocturia. On day 4 , the patient had an altered state of consciousness and an episode of atrial fibrillation, which was assessed and reversed. Since there was a risk of central nervous system (CNS) thrombosis, anticoagulation was prescribed. A computed tomography (CT) scan of the head showed an old lacunar infarction on the left superior cerebellar artery and chronic ischemic microangiopathy. On day 7 , 
hypoxia (85\%) and another episode of atrial fibrillation occurred. Chest radiography showed opacification of the left hemithorax, the cardiac silhouette was not visible and prominent pulmonary hilar vessels with atelectasis on the right apical lobe were observed. Considering the size of the pleural effusion, the patient's condition and the associated risks, on day 8 , the patient was referred to an intermediate care unit in a tertiary-level hospital, in the same city.

On day 9, the blood count showed decreased number of erythrocytes ( 2.54 million cells $/ \mu \mathrm{L})$ with low hematocrit $(23.7 \%)$ and hemoglobin $(8.6 \mathrm{~g} / \mathrm{dL})$. Platelets and leukocytes counts were normal, although there was an increased percentage of neutrophils (78\%). The blood chemistry revealed normal levels of electrolytes, blood urea nitrogen and creatinine. On the same day, a closed tube thoracostomy was conducted and $2,500 \mathrm{~mL}$ of pleural fluid, of bloody appearance, were drained. The pleural fluid had normal $\mathrm{pH}$, glucose and albumin, with low protein $(1.9 \mathrm{~g} / \mathrm{L})$ and high lactate dehydrogenase $(2,437$ IU/L). The leukocyte count was very low $(23$ cells $/ \mathrm{mL})$ with $80 \%$ polymorphonuclear and $20 \%$ mononuclear cells. The erythrocyte count was elevated $(13,498$ cells $/ \mu \mathrm{L})$ with $95 \%$ biconcave and $5 \%$ crenated cells. Altogether, the characteristics of the pleural fluid suggested an inflammatory process. No microorganisms were observed with Gram and ZiehlNeelsen staining of the pleural fluid. PCR for mycobacteria and cultures for bacteria and fungi of the pleural fluid were negative. India ink was not performed on the pleural fluid as there was not suspicion of cryptococcal infection.

On day 12, the patient was transferred to a general ward. Two days later, chest and abdominal CT scans confirmed the pleural effusion on the left side (Figure 1) and showed an abdominal mass and renal atrophy on the left kidney, suggesting a metastatic pleural neoplasm. Blood cultures were performed and pleural biopsies were taken on days 16 and 18, respectively. However, the patient's condition deteriorated, and the woman died after 21 days of hospitalization. Pleural biopsies reported a chronic nonspecific pleuritis. Cryptococcus albidus was recovered from the blood culture. The isolate was identified by the VITEK $^{\otimes 2}$ YST ID card (bioMérieux, France) and confirmed by MALDI-TOF mass spectrometry with the MYLA $^{\circledR}$ software $\left(\right.$ VITEK $^{\circledR}$ MS, bioMérieux, France), with an acceptable confidence value of $99.9 \%$. Thus, cryptococcemia was diagnosed.

\section{DISCUSSION}

This report presents a case of lethal fungaemia, with involvement of the pleura, caused by a non-C. neoformans cryptococcal species, an extremely rare presentation, whose

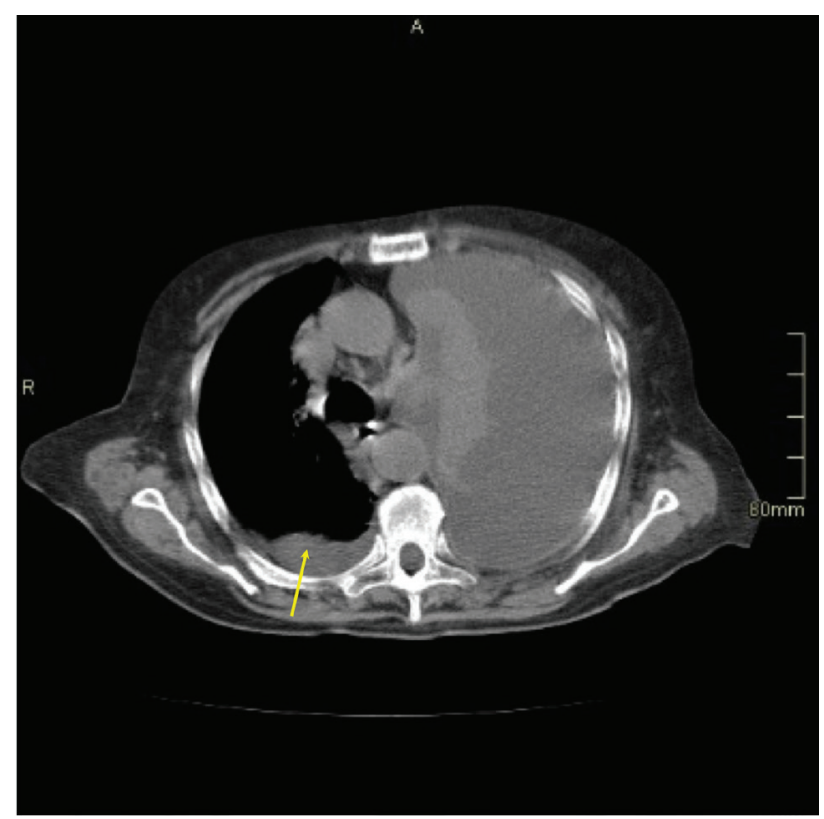

Figure 1 - Computed tomography scan of the chest showing a massive left-side pleural effusion with a compressive atelectasis in the right apical lobe (arrow).

diagnosis can be easily delayed or mistaken. As the lungs are the primary target organs of cryptococcal species, pulmonary disease is still an important infection that may be underdiagnosed due to the nonspecific symptoms and broadly categorized findings. Predisposing factors such as hematologic malignancies, lung cancer, solid organ transplantation, diabetes mellitus, glucocorticosteroid use, and hepatic cirrhosis increase the risk for developing pulmonary cryptococcosis, which may manifest as pulmonary nodules, masses or infiltrates, mediastinal lymphadenopathy or less frequently pleural effusions ${ }^{3}$. However, in a recent review, only 50 cases of pleural effusion, as a manifestation of pulmonary cryptococcosis, have been reported ${ }^{4}$. Even rarer are cryptococcal infections of the pleura caused by non-C. neoformans species. Nonetheless, this number may be underestimated because in many cases, as occurred in the case described herein, cultures from the pleural fluid are generally negative for Cryptococcus spp., making diagnosis even more challenging. Additionally, direct microscopy to observe yeast-like cells in the pleural fluid is not always possible, due mainly to the small amount of microorganisms present in the fluid ${ }^{5,6}$.

Similarly to C. neoformans and its sibling species $C$. gattii, the main etiological agents of cryptococcosis, C. albidus is a free-living encapsulated yeast that resides in the environment and whose clinical relevance remains unknown ${ }^{7}$, though with an incremental rise in the number of at-risk patients, the incidence of infections due to non- $C$. neoformans/ non $C$. gattii species have also increased, with $C$. albidus 
and C. laurentii accounting for $80 \%$ of reported cases $^{8-10}$. Among the few cases of cryptococcal infection involving the pleural space, only one has been reported to be caused by $C$. albidus, although concomitantly with mucormycosis and in a patient undergoing hemodialysis ${ }^{11}$. Particularly for C. albidus, bloodstream and CNS are the commonest sites of infection $^{8,12}$. In addition, until now in Colombia, C. albidus has not been reported as a causative agent of disease and its isolation from environmental sources has been rather sporadic $^{13,14}$.

While in many patients without predisposing condition, pleural effusion presents only as localized disease ${ }^{6,15}$, in the current case, positive blood culture for C. albidus indicated that the pleural effusion has possibly led to the dissemination of the microorganism. This case of an elderly woman who succumbed due to cryptococcal pleuritis highlights the importance of considering not only the risk factors of the patients, but also the emergence of new pathogens, requiring an appropriate and prompt diagnosis. In comparison with other cases of cryptococcal pleural infection, the case reported here was difficult to diagnose because of the variety of general signs and symptoms of the patient, her chronic condition of T2DM and advanced age. Initially, pleural tuberculosis was considered, then, the bloody appearance of the pleural fluid suggested metastatic pleural neoplasms or metastatic malignancies, and the onset of neurological symptoms also biased the diagnosis. Only post-mortem, fungaemia and disseminated cryptococcosis were diagnosed.

Even though $C$. albidus was correctly identified, its identification was only possible with automatized methodologies, namely VITEK ${ }^{\circledR} 2$ YST ID system and MALDI-TOF MS. Morphologically and physiologically, species within the genus Cryptococcus are very easily mistaken, for instance, serotyping of some C. albidus strains leads to grouping them as $C$. neoformans, serotype A strains ${ }^{7}$. However, C. neoformans differs from C. albidus by the activity of the phenoloxidase enzyme that allows it to synthesizemelanin. Growth of brown to black pigmented colonies on specific media allows the differentiation of C. neoformans and C. gattii, from other yeasts of the genus, including C. albidus. C. albidus does not produce melanin due to the lack of phenoloxidase activity ${ }^{7}$, which helps the diagnosis (Figure 2).

To conclude, this case emphasizes the importance of considering atypical infections like cryptococcal infection of the pleura in the differential diagnosis of patients with pleural effusion. In addition, it underlines the capacity of $C$. albidus to cause disseminated disease, which is uncommon, but is a very serious condition that should be deemed possible in diabetic patients and in the elderly and critically ill individuals. As reported here, the non-specific
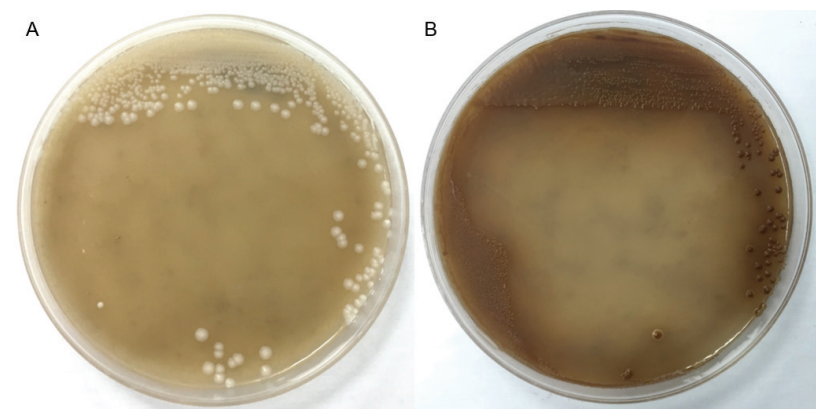

Figure 2 - Cryptococcus albidus (A) and Cryptococcus neoformans (B) grown in Niger seed agar. $C$. albidus colonies grow cream-colored, while $C$. neoformans colonies produce melanin, due to the phenoloxidase activity, and turn brown.

clinical features of pulmonary disease can hinder an opportune treatment, leading to an increased morbidity and even in some cases increased mortality rates. Thus, the correct and prompt identification and the surveillance of non-prevalent fungal species, such as C. albidus, causing disease in chronically ill individuals, will aid determining the real impact of these atypical fungi as etiologic agents of disseminated diseases. Additionally, the surveillance of antifungal susceptibility of emerging pathogens is recommended to help choosing specific management for individual patients, considering that species like C. albidus are less susceptible or even resistant to azoles ${ }^{7}$, one of the most commonly used drugs for the treatment of cryptococcosis and other mycoses. C. albidus must be then added to the growing number of recently described causative agents of disseminated mycoses in diabetic patients.

\section{AUTHORS' CONTRIBUTIONS}

All authors contributed with the preparation and edition of the manuscript.

\section{CONFLICT OF INTERESTS}

The authors declare that this work does not have any commercial or financial relationship that could be considered a potential conflict of interest.

\section{ETHICAL STATEMENT}

The work has been approved by the Ethics in Research Committee from the Universidad del Rosario (DVO005 845-CV1128).

\section{FUNDING}

No funding agencies had a role in the preparation, review or approval of the manuscript. 


\section{REFERENCES}

1. Rajasingham R, Smith RM, Park BJ, Jarvis JN, Govender NP, Chiller TM, et al. Global burden of disease of HIV-associated cryptococcal meningitis: an updated analysis. Lancet Infect Dis. 2017; 17:873-81.

2. Pappas PG. Cryptococcal infections in non-HIV-infected patients. Trans Am Clin Climatol Assoc. 2013;124:61-79.

3. Chang CC, Sorrell TC, Chen SC. Pulmonary cryptococcosis. Semin Respir Crit Care Med. 2015;36:681-91.

4. Wee AC, Seet JE, Venkatalacham J, Tan SK. Cryptococcal pleural infection in a recurrent pleural effusion: a case report. Respirol Case Rep. 2018;6:e00294.

5. Shankar EM, Kumarasamy N, Bella D, Renuka S, Kownhar $\mathrm{H}$, Suniti S, et al. Pneumonia and pleural effusion due to Cryptococcus laurentii in a clinically proven case of AIDS. Can Respir J. 2006;13:275-8.

6. Young EJ, Hirsh DD, Fainstein V, Williams TW. Pleural effusions due to Cryptococcus neoformans: a review of the literature and report of two cases with cryptococcal antigen determinations. Am Rev Respir Dis. 1980;121:743-7.

7. Fonseca AJ, Fell JW, Boekhout T. Cryptococcus Vuillemin. In: Kurtzman C, Fell JW, Boekhout T, editors. The yeast: a taxonomic study. $5^{\text {th }}$ ed. Amsterdam: Elsevier; 2010. p.1665740.
8. Choe YJ, Blatt DB, Yalcindag A, Geffert SF, Bobenchik AM, Michelow IC. Cryptococcus albidus fungemia in an immunosuppressed child: case report and systematic literature review. J Pediatric Infect Dis Soc. 2019;9:100-5.

9. Khawcharoenporn T, Apisarnthanarak A, Mundy LM. Nonneoformans cryptococcal infections: a systematic review. Infection. 2007;35:51-8.

10. Smith N, Sehring M, Chambers J, Patel P. Perspectives on non-neoformans cryptococcal opportunistic infections. J Community Hosp Intern Med Perspect. 2017;7:214-7.

11. Horowitz ID, Blumberg EA, Krevolin L. Cryptococcus albidus and mucormycosis empyema in a patient receiving hemodialysis. South Med J. 1993;86:1070-2.

12. Aghaei Gharehbolagh S, Nasimi M, Agha Kuchak Afshari S, Ghasemi Z, Rezaie S. First case of superficial infection due to Naganishia albida (formerly Cryptococcus albidus) in Iran: a review of the literature. Curr Med Mycol. 2017;3:33-7.

13. Callejas A, Ordoñez N, Rodriguez MC, Castañeda E. First isolation of Cryptococcus neoformans var. gattii, serotype $\mathrm{C}$, from the environment in Colombia. Med Mycol. 1998;36:341-4.

14. Castañeda A, Castañeda E. Aislamiento de especies de Cryptococcus asociadas con Eucalyptus en un parque de Bogotá. Biomedica. 2001;21:75-8.

15. Rodríguez-Álvarez A, Fernández-Rial Á, Pérez-López A, Pita J. Cryptococcus neoformans pleuritis in an immunocompetent patient. Arch Bronconeumol. 2020;56:59-60. 